\title{
Boundary Conditions for the Solution of Compressible Navier-Stokes Equations by an Implicit Factored Method
}

\author{
ToM I-P. SHIH* \\ NASA-Lewis Research Center, Cleveland, Ohio 44135 \\ Gene E. Smith and George S. Springer \\ Department of Mechanical Engineering and Applied Mechanics, \\ The University of Michigan, Ann Arbor, Michigan 48109
}

\author{
AND \\ Y. RIMON \\ Computer Science Department, Computing Center, \\ Department of Defense, Haifa, Israel \\ Received October 13, 1982; revised January 13, 1983
}

\begin{abstract}
A method is presented for formulating the boundary conditions in implicit finite-difference form needed for obtaining solutions to the compressible Navier-Stokes equations by the Beam and Warming implicit factored method. The usefulness of the method was demonstrated (a) by establishing the boundary conditions applicable to the analysis of the flow inside an axisymmetric piston-cylinder configuration and (b) by calculating velocities and mass fractions inside the cylinder for different geometries and different operating conditions. Stability, selection of time step and grid sizes, and computer time requirements are discussed in reference to the piston-cylinder problem analyzed.
\end{abstract}

\section{INTRODUCTION}

Considerable efforts have been made in recent years to develop numerical methods which permit solutions to complex flow problems. One such method was recently proposed by Beam and Warming $[1,2]$. This method, referred to as the implicit factored method, has a number of attractive features, including unconditional linear stahility, efficient noniterative solution procedure, applicability to the study of steady and unsteady one-, two-, and three-dimensional flows, and compatibility with parallel and vector processors.

* Now at University of Florida, Department of Mechanical Engineering, Gainesville, Fla. 32611. 


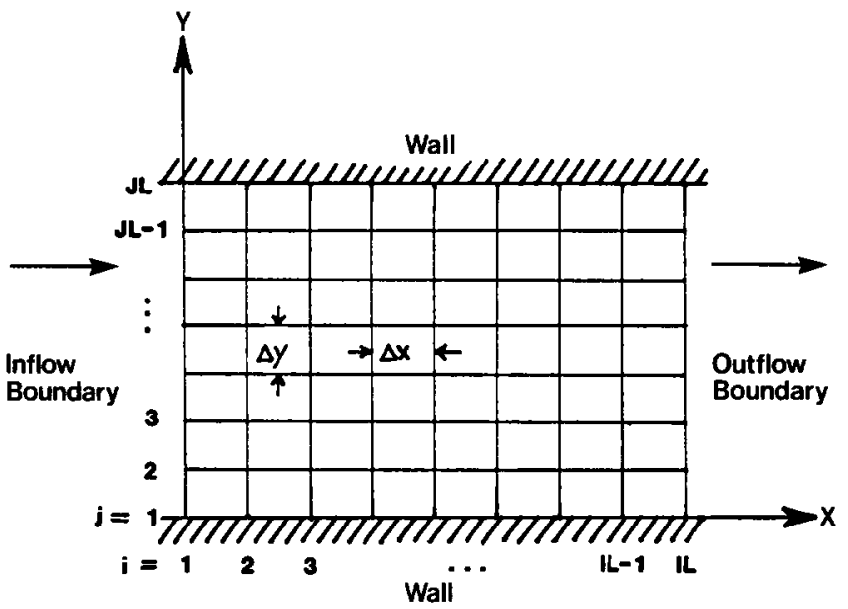

Fig. 1. Computational domain and grid system.

In order to realize the full potential of the implicit factored method, the boundary conditions must also be expressed in implicit finite-difference form (hereafter referred to as implicit boundary conditions) [2-4]. In this paper, a method is described which can be used to express boundary conditions in implicit form for every possible type of boundary including stationary or moving, impermeable or permeable walls, and open surfaces across which flow occurs (inflow and outflow boundaries). In the interest of brevity, the procedures are described in detail for two types of boundaries, namely, for flow adjacent to a stationary impermeable wall and for subsonic flow across an outflow boundary. However, it is explained how the results can readily be extended to other types of boundaries.

For illustrative purposes, the method is presented in Cartesian coordinates for twodimensional, nonreacting laminar flow problems involving rectangular boundaries (Fig. 1). The method could readily be extended to other types of two- and threedimensional problems and to turbulent, chemically reacting flows.

In order to demonstrate the usefulness of the procedure devised for obtaining implicit boundary conditions, solutions where obtained showing the velocity field and the specie mass fractions inside an axisymmetric piston-cylinder configuration during the intake and compression strokes. Stability, selection of time step and grid sizes, and computer time requirements are discussed in reference to the sample solutions.

\section{Governing Equations}

The governing equations of compressible laminar flows of viscous, thermally conducting, nonreacting, two-component gas mixtures are summarized in Table I [5]. The equations presented in this table apply to two-dimensional problems and include 
TABLE I

Governing Equations ${ }^{a}$

Equation

Eq. No.

$$
\begin{aligned}
& \frac{\partial \rho}{\partial t}+\frac{\partial \rho V_{x}}{\partial x}+\frac{\partial \rho V_{y}}{\partial y}=0 \\
& \frac{\partial \rho X_{q}}{\partial t}+\frac{\partial}{\partial x} \rho X_{A}\left(V_{x}+V_{A X}\right)+\frac{\partial}{\partial y} \rho X_{A}\left(V_{y}+V_{A y}\right)=0 \\
& \frac{\partial \rho V_{x}}{\partial t}+\frac{\partial}{\partial x}\left(\rho V_{x}^{2}\right)+\frac{\partial}{\partial y}\left(\rho V_{x} V_{y}\right)=-\frac{\partial P}{\partial x}+\frac{\partial \tau_{x x}}{\partial x}+\frac{\partial \tau_{y x}}{\partial y} \\
& \frac{\partial \rho V_{y}}{\partial t}+\frac{\partial}{\partial x}\left(\rho V_{x} V_{y}\right)+\frac{\partial}{\partial y}\left(\rho V_{y}^{2}\right)=-\frac{\partial P}{\partial y}+\frac{\partial \tau_{x y}}{\partial x}+\frac{\partial \tau_{y y}}{\partial y} \\
& \frac{\partial e}{\partial t}+\frac{\partial}{\partial x}(e+P) V_{x}+\frac{\partial}{\partial y}(e+P) V_{y}=\frac{\partial}{\partial x}\left(\tau_{x x} V_{x}+\tau_{x y} V_{y}\right) \\
& \quad+\frac{\partial}{\partial y}\left(\tau_{x y} V_{x}+\tau_{y y} V_{y}\right)-\frac{\partial}{\partial x} q_{x}-\frac{\partial}{\partial y} q_{y}
\end{aligned}
$$

where

$$
\begin{aligned}
& V_{A x}=-\frac{D_{A B}}{X_{A}} \frac{\dot{c}}{\partial x} X_{A}=-\left(c / p X_{4}\right) \frac{\hat{c}}{\partial x} X_{4} \\
& V_{A y}=-\frac{D_{A B}}{x_{A}} \frac{\dot{c}}{\partial y} X_{A}=-\left(c / \rho X_{A}\right) \frac{\partial}{\partial y} x_{A} \\
& \tau_{x x}=2 \mu \frac{\partial V_{x}}{\partial x}-\frac{2}{3} \mu\left(\frac{\partial V_{x}}{\partial x}+\frac{\partial V_{y}}{\partial y^{\prime}}\right) \\
& \tau_{y y}=2 \mu \frac{\partial V_{y}}{\partial y^{\prime}}-\frac{2}{3} \mu\left(\frac{\partial V_{x}}{\partial x}+\frac{\partial V_{y}}{\partial y}\right) \\
& \tau_{x y}-\mu\left(\frac{\partial V_{x}}{\partial y}+\frac{\partial V_{y}}{\partial x}\right) \\
& h_{A}=h_{A}^{\circ}+\int_{T_{i}}^{T} c_{P A} d T=h_{A}^{\circ}+c_{P A}\left(T-T_{i}\right) \\
& h_{B}=h_{B}^{\circ}+\int_{T_{i}}^{T} c_{P B} d T=h_{B}^{\circ}+C_{P B}\left(T-T_{i}\right) \\
& q_{x}=-\lambda \frac{\partial T}{\partial x}+\rho X_{A} V_{A x}\left(h_{A}-h_{B}\right)=-\lambda \frac{\partial T}{\partial x}-c\left[\left(h_{A}^{\circ}-h_{B}^{\circ}\right)+\left(c_{p_{A}}-C_{P_{B}}\right)\left(T-T_{i}\right) \mid \frac{\dot{c}}{\partial x} X_{A}\right. \\
& q_{y}=-\lambda \frac{\partial T}{\partial y}+\rho X_{A} V_{A y}\left(h_{A}-h_{B}\right)=-\lambda \frac{\partial T}{\partial y}-c\left[\left(h_{A}^{\circ}-h_{B}^{\circ}\right)+\left(c_{P_{A}}-C_{P A}\right)\left(T-T_{i}\right)\right] \frac{\partial}{\partial y} X_{A} \\
& X_{B}=1-X_{A} \\
& P=\rho R T\left(\frac{X_{A}}{M_{A}}-\frac{X_{B}}{M_{B}}\right)=\rho R T\left[\left(\frac{1}{M_{A}}-\frac{1}{M_{B}}\right) X_{4}+\frac{1}{M_{B}}\right] \\
& e=\rho\left[\left(h_{A} X_{A}+h_{B} X_{B}\right)-\frac{P}{\rho}+\frac{1}{2}\left(V_{x}^{2}+V_{y}^{2}\right)\right] \\
& =\rho X_{A}\left[\left(h_{4}^{\circ}-h_{B}^{\circ}\right)-\left(C_{P_{A}}-C_{P B}\right) T_{i}\right]+\rho\left(h_{B}^{\circ}-C_{P B} T_{i}\right) \\
& +\frac{1}{2} \rho\left(V_{x}^{2}+V_{y}^{2}\right)-P+\rho\left[\left(C_{P_{A}}-C_{P_{B}}\right) X_{A}+C_{P B}\right] T
\end{aligned}
$$

${ }^{a} C_{p i}=$ Constant pressure specific heat of component $i, D_{A B}=$ binary diffusion coefficient for components $A$ and $B, e=$ energy per unit volume, $h_{i}=$ specific enthalpy of component $i, h_{i}^{\circ}=$ standard enthalpy of formation per unit mass for component $i$ at temperature $T_{i}, M_{i}=$ molecular weight of component $i, P=$ static pressure, $q_{j}=$ heat flux in the $j$-direction, $R=$ universal gas constant, $V_{j}=$ $j$-component of the velocity, $V_{i j}=j$-component of the diffusion velocity for component $i, X_{i}=$ mass fraction of component $i, \lambda=$ thermal conductivity, $\mu=$ viscosity, $\rho=$ density, $\tau=$ shear stress. 
the following simplifications: (i) all thermodynamic and transport properties (except for the binary diffusion coefficient) are constants; (ii) the binary diffusion coefficient between the two-components of the mixture is inversely proportional to the local mixture density; (iii) pressure gradient contribution to specie diffusion and momentum transport due to specie diffusion are negligible; (iv) irreversible coupling effects between the temperature gradient and specie concentration gradients (Soret and Dufour effects) are negligible; (v) body forces are small compared to inertia forces; (vi) bulk viscosity of the gases is zero; and (vii) radiation heat transfer is negligible.

Equations (1)-(17) (Table I) constitute a closed system in five dependent variables: density $(\rho)$, mass fraction of component $A\left(X_{A}\right), x$-component of the velocity $\left(V_{x}\right), y$-component of the velocity $\left(V_{y}\right)$, and energy $(e)$.

In order to obtain numerical solutions to the governing equations, the spatial domain is represented by a grid system in which the grid points (denoted by subscripts $i$ and $j$ ) are uniformly distributed (Fig. 1). For convenience, the grid points are divided into two groups. In the first group are the interior grid points $(i=2,3, \ldots$, $I L-1 ; j=2,3, \ldots, J L-1)$. In the second group are the boundary grid points $(i=1, \quad j=1,2, \ldots, J L ; \quad i=I L, \quad j=1,2, \ldots, J L ; j=1, \quad i=2,3, \ldots, I L-1 ; j=J L$, $i=2,3, \ldots, I L-1)$.

The solution (i.e., the values of the dependent variables at every grid point) is assumed to be known at time $t$ and $t-\Delta t$ (denoted by superscripts $n$ and $n-1$, respectively). The solution at time $t+\Delta t$ is sought.

By replacing the time derivatives in the governing equations by a time-difference formula, by linearizing terms which are nonlinear functions of unknown dependent variables (i.e., dependent variables at time $t+\Delta t$ ), and by employing an approximate factorization procedure, the governing equations (Eqs. (1)-(5)) applied at every grid point can be written in the form $[1,2,6]$

$$
\left(1+\frac{\Theta \Delta t}{1+\gamma} \mathbf{X}_{i, j}^{n}\right)\left(\mathbf{I}+\frac{\Theta \Delta t}{1+\gamma} \mathbf{Y}_{i, j}^{n}\right) \Delta \mathbf{U}_{i, j}^{n+1}=\mathbf{R}_{i, j}^{n}
$$

In the above equation, $\Delta t$ is the time step size. $\Theta$ and $\gamma$ are constants which determine the type of time-difference formula to be used. In this study, $\Theta$ and $\gamma$ were set equal to one and one-half, respectively, resulting in the three-point backward implicit formula. $\mathbf{I}$ is a five-by-five identity matrix. $\Delta \mathbf{U}_{i, j}^{n+1}$ and $\mathbf{R}_{i, j}^{n}$ are fivecomponent vectors. $\mathbf{X}_{i, j}^{n}$ and $\mathbf{Y}_{i, j}^{n}$ are five-by-five matrices whose elements are $x$ derivative and $y$-derivative operators, respectively. The elements of $\mathbf{R}_{i, j}^{n}, \mathbf{X}_{i, j}^{n}$, and $\mathbf{Y}_{i, j}^{n}$ are all expressed in terms of known quantities (i.e., in terms of the dependent variables at time $t$ and $t-\Delta t$ ). The details of the elements of $\boldsymbol{R}_{i, j}^{n}, \mathbf{X}_{i, j}^{n}$, and $\mathbf{Y}_{i, j}^{n}$ are not germane to the procedure for deriving the implicit boundary conditions. Hence they are not given here, but can be found in Refs. $[1,2,6]$. The elements of $\Delta \mathbf{U}^{n+1}$ contain the unknown dependent variables being sought

$$
\Delta \mathbf{U}^{n+1}=\mathbf{U}^{n+1}-\mathbf{U}^{n},
$$


where

$$
\mathbf{U}=\left[\begin{array}{l}
\rho \\
\rho X_{\mathrm{A}} \\
\rho V_{x} \\
\rho V_{y} \\
e
\end{array}\right]=\left[\begin{array}{l}
\rho \\
x \\
m \\
n \\
e
\end{array}\right]
$$

The delta operator $\Delta()^{n+1}$ is defined as

$$
\Delta()^{n+1}=()^{n+1}-()^{n} .
$$

Equation (18) is second-order accurate in time $[1,2,6]$. Therefore, all approximations introduced in the subsequent solution procedure will also be second-order accurate in time.

Equation (18) may be expressed in either of the following two ways:

$$
\begin{gathered}
\left(1+\frac{\Theta \Delta t}{1+\gamma} \mathbf{X}_{i, j}^{n}\right) \Delta \mathbf{U}_{i, j}^{*}=\mathbf{R}_{i, j}^{n}, \\
\left(1+\frac{\Theta \Delta t}{1+\gamma} \mathbf{Y}_{i, j}^{n}\right) \Delta \mathbf{U}_{i, j}^{n+1}=\Delta \mathbf{U}_{i, j}^{*},
\end{gathered}
$$

or

$$
\begin{gathered}
\left(1+\frac{\Theta \Delta t}{1+\gamma} \mathbf{Y}_{i, j}^{n}\right) \Delta \mathbf{U}_{i, j}^{*}=\mathbf{R}_{i, j}^{n}, \\
\left(1+\frac{\Theta \Delta t}{1+\gamma} \mathbf{X}_{i, j}^{n}\right) \Delta \mathbf{U}_{i, j}^{n+1}=\Delta \mathbf{U}_{i, j}^{*} .
\end{gathered}
$$

$\Delta \mathbf{U}_{i, j}^{*}$, a five-component vector, is an intermediate variable defined by either Eq. (23) or Eq. (25). The five rows of each of the above four equations correspond to the conservation equations of mass, specie, $x$ momentum, $y$ momentum, and energy, respectively.

Either set of the "split" equations (i.e., Eqs. (22) and (23) or Eqs. (24) and (25)) may be used in the solution. In this paper, Eqs. (24) and (25) were adopted. Solutions to Eqs. (24) and (25) together with the boundary conditions developed in the next section provide the unknown dependent variable $\Delta \mathbf{U}^{n+1}$.

To generate solutions, the governing equations (Eqs. (24) and (25)) must be expressed in finite-difference form. The finite-difference form of these equations suitable for solution with the efficient Thomas algorithm were presented by Beam and Warming $[1,2]$. 


\section{Boundary Conditions}

To proceed with the solution of Eqs. (24) and (25), expressions for $\Delta \mathrm{U}^{*}$ are needed at the two parallel boundaries perpendicular to the $y$-axis $(j=1, i=2,3, \ldots$, $I L-1$ and $j=J L, i=2,3, \ldots, I L-1)$ and expressions for $\Delta \mathbf{U}^{n+1}$ are needed at the other two parallel boundaries $(i=1, j=2,3, \ldots, J L-1$ and $i=I L, j=2,3, \ldots, J L-1$; Fig. 1).

It is desired to develop expressions for $\Delta \mathbf{U}^{*}$ and $\Delta \mathbf{U}^{n+1}$ at the boundary grid points which, when combined with the finite-difference form of Eqs. (24) and (25), produce systems of equations with block tridiagonal coefficient matrices. These systems of equations can then be solved by the highly efficient Thomas algorithm. At the boundary grid points, the most general expressions for $\Delta \mathbf{U}^{*}$ and $\Delta \mathbf{U}^{n+1}$ which permit the use of the Thomas algorithm are [6]

$$
\begin{aligned}
\Delta \mathbf{U}_{i, S}^{*} & =\mathbf{a}_{i}+\mathbf{b}_{i} \Delta \mathbf{U}_{i, S 1}^{*}+\mathbf{c}_{i} \Delta \mathbf{U}_{i, S 2}^{*}, \\
\Delta \mathbf{U}_{T, j}^{n+1} & =\mathbf{d}_{j}+\mathbf{e}_{j} \Delta \mathbf{U}_{T 1, j}^{n+1}+\mathbf{f}_{j} \Delta \mathbf{U}_{T, j}^{n+1} .
\end{aligned}
$$

The subscripts $S, S 1$, and $S 2$ represent either 1,2 , and 3 or $J L, J L-1$, and $J L-2$ for the two boundaries perpendicular to the $y$-axis. The subscripts $T, T 1$, and $T 2$ represent either 1,2 , and 3 or $I L, I L-1$, and $I L-2$ for the two boundaries perpendicular to the $x$-axis. $\mathbf{a}_{i}$ and $\mathbf{d}_{j}$ are five-component vectors. $\mathbf{b}_{i}, \mathbf{c}_{i}, \mathbf{e}_{j}$, and $\mathbf{f}_{j}$ are five-by-five matrices. The elements of $\mathbf{a}_{i}, \mathbf{b}_{i}, \mathbf{c}_{i}, \mathbf{d}_{j}, \mathbf{e}_{j}$, and $\mathbf{f}_{j}$ depend on known quantities $\mathbf{U}^{n}$ and $\mathbf{U}^{n-1}$ in an as yet unknown manner. The objective is to determine $\mathbf{a}_{i}, \mathbf{b}_{i}, \mathbf{c}_{i}, \mathbf{d}_{j}, \mathbf{e}_{j}$, and $\mathbf{f}_{j}$.

When the dependent variables $\left(\rho, X_{\mathrm{A}}, V_{x}, V_{y}\right.$, and $\left.e\right)$ are all constant along the boundary, it can be shown that [6]

$$
\begin{aligned}
\mathbf{a}_{i} & =\mathbf{d}_{j}=0, \\
\mathbf{b}_{i} & =\mathbf{c}_{i}=\mathbf{e}_{j}=\mathbf{f}_{j}=0 .
\end{aligned}
$$

In practical problems, $\mathbf{a}_{i}, \mathbf{b}_{i}, \mathbf{c}_{i}, \mathbf{d}_{j}, \mathbf{e}_{j}$, and $\boldsymbol{f}_{j}$ are not zero. The procedure proposed in this study to determine $\mathbf{a}_{i}, \mathbf{b}_{i}$, and $\mathbf{c}_{i}$ involves the following major steps:

(i) $\Delta \mathbf{U}^{*}$ is expressed in terms of $\Delta \mathbf{U}^{n+1}$ at the boundary.

(ii) $\Delta \mathbf{U}^{n+1}$ at the boundary is expressed in terms of $\Delta \mathbf{U}^{n+1}$ at adjacent interior grid points.

(iii) $\Delta \mathbf{U}^{n+1}$ is expressed in terms of $\Delta \mathbf{U}^{*}$ at corresponding interior grid points.

The determination of $\mathbf{d}_{j}, \boldsymbol{e}_{j}$, and $\mathbf{f}_{j}$ involve only step (ii) above.

The above procedure is general and is applicable to different types of boundaries. In Sections 3.1 to 3.3, the details of the approach are given for two types of boundaries, namely, for flow adjacent to a stationary, impermeable wall and for subsonic flow across an outflow boundary. By following the procedure given for these two examples, the method can readily be extended to other types of boundaries. 
It is noted here that Thomas [7] has also proposed a method for deriving implicit boundary conditions for the implicit factored method. The major difference between the method presented here and that of Thomas is in the procedure employed to derive an expression for the intermediate variable $\Delta \mathbf{U}^{*}$ at the boundary. Thomas' procedure is based on an assumption regarding the relationship between the elements in $\Delta \mathbf{U}^{*}$. Thomas assumed that the relationship between the elements in $\Delta \mathbf{U}^{*}$ is the same as the relationship between the elements in $\Delta \mathbf{U}^{n+1}$. This assumption was not made in the present analysis.

\subsection{Physical Boundary Conditions}

Derivation of expressions for $\Delta \mathbf{U}^{*}$ and $\Delta \mathbf{U}^{n+1}$ at the boundary requires a knowledge of the physical boundary conditions. As noted previously, in this paper we are concerned with the detailed development of implicit boundary conditions for two types of boundaries, namely, stationary, impermeable wall and outflow boundary with locally subsonic flow. At a stationary, impermeable wall, the following conditions (physical boundary conditions) must be satisfied: II).

(i) The gas velocity is equal to the wall velocity (Eqs. (29) and (30), Table

(ii) There is no mass diffusion into the wall (Eq. (31), Table II).

(iii) The gas temperature is equal to the wall temperature (Eq. (32), Table II). If the wall temperature is unknown, then the heat flux through the wall must be given (Eq. (33), Table II).

The outflow boundary is an imaginary surface (defined arbitrarily to specify a finite computational domain) which cannot produce boundary layers $|8,9|$. The physical conditions which must be satisfied at an outflow boundary depend on whether the flow is locally subsonic or supersonic. For subsonic flow with the flow parallel to the $x$-axis, the following conditions have been shown to be reasonable and useful at the outflow boundary $[9,10]$ :

(i) The static pressure is equal to the back pressure $P_{\mathrm{b}}$ (Eq. (34), Table II).

(ii) The second-order derivative of mass fraction $X_{\mathrm{A}}$ along the flow direction is equal to zero (Eq. (35), Table II).

(iii) The second-order derivative of the velocity along the flow direction is equal to zero (Eqs. (36) and (37), Table II).

Conditions other than those given above may be specified at the outflow boundary $[3,11-13]$.

\subsection{Expressions for $\Delta \mathrm{U}^{*}$ at the Boundary}

For the problem depicted in Fig. 1, expressions for $\Delta \mathrm{U}^{*}$ are needed at the upper and the lower walls. Here only the derivation of the expression applicable at the upper wall $(j=J L, i=1,2, \ldots, I L)$ is presented. 
TABLE II

Physical Bondary Conditions ${ }^{a}$

\begin{tabular}{ccc}
\hline $\begin{array}{c}\text { Type of } \\
\text { buundary }\end{array}$ & Equation & Eq. No. \\
\hline $\begin{array}{c}\text { Impermeable } \\
\text { wall }\end{array}$ & $V_{x}=\left(V_{\mathrm{w}}\right)_{x}$ \\
$V_{y}=\left(V_{\mathrm{w}}\right)_{y}$ & \\
$\frac{\partial}{\partial \eta} X_{4}=0$ & \\
$T=T_{\mathrm{w}}$ & \\
$q_{\mathrm{w}}=-\lambda \frac{\partial}{\partial \eta} T$ \\
$\begin{array}{c}\text { Subsonic } \\
\text { outflow }\end{array}$ \\
$\frac{\partial^{2}}{\partial \xi^{2}} X_{4}=0$ \\
$\frac{\partial^{2}}{\partial \xi^{2}} V_{x}=0$ \\
$\frac{\partial^{2}}{\partial \xi^{2}} V_{y}=0$
\end{tabular}

${ }^{a} \partial / \partial \eta=$ Normal derivative at the wall, $\partial^{2} / \partial \xi^{2}=$ derivative along the flow direction. $\left(V_{\mathrm{w}}\right)_{x}$ and $\left(V_{\mathrm{w}}\right)_{y}=x$ - and $y$-component of the wall velocity, $T_{\mathrm{w}}=$ wall temperature. $q_{\mathrm{w}}=$ heat flux at wall.

\subsection{1. $\Delta \mathbf{U}^{*}$ as a Function of $\Delta \mathbf{U}^{n+1}$ at the Boundary}

In general, $\Delta \mathbf{U}^{*}$ is related to $\Delta \mathbf{U}^{n+1}$ through Eq. (25). However, when an element of $\Delta \mathbf{U}^{n+1}$ is zero, the corresponding element in $\Delta \mathbf{U}^{*}$ is also zero [6]. Formulation of the present problem requires only $\Delta \mathbf{U}^{*}$ at walls. At these walls, the $x$ and $y$ velocity components are zero (Eqs. (29) and (30)) and $\Delta \mathbf{U}^{n+1}$ at the upper wall $(j=J L$, $i=1,2, \ldots, I L)$ is

$$
\Delta \mathbf{U}_{i, J L}^{n+1}=\left[\begin{array}{c}
\Delta \rho \\
\Delta x \\
0 \\
0 \\
\Delta e
\end{array}\right]_{i, J L}^{n+1}
$$


Equation (25) at the upper wall becomes

$$
\begin{aligned}
\Delta \mathbf{U}_{i, J L}^{*} & =\left(1+\frac{\Theta \Delta t}{1+\gamma} \mathbf{X}_{i, J L}^{n}\right) \Delta \mathbf{U}_{i, J L}^{n+1} \\
& =\Delta \mathbf{U}_{i, J L}^{n+1}+\frac{\Theta \Delta t}{1+\gamma} \mathbf{X}_{i, J L}^{n} \Delta \mathbf{U}_{i, J L}^{n+1} .
\end{aligned}
$$

It is important that the third and fourth rows of Eq. (39) which correspond to the $x$ and $y$ momentum equations be set equal to zero [6].

The second term on the right-hand side of Eq. (39) includes $x$-derivatives (recall that $\mathbf{X}_{i, j}^{n}$ is an $x$ differential operator). Calculation of these derivatives requires knowledge of $\Delta \mathbf{U}^{*}$ at adjacent lines having different $x$ values (e.g., $\Delta \mathbf{U}_{i+1, j l .}^{*}$ and $\left.\Delta \mathrm{U}_{i-1, J L}^{*}\right)$. To avoid the necessity of calculating unknowns at more than one $x$ line at a time, $\Delta \mathbf{U}^{n+1}$ in the second term of Eq. (39) is approximated by an expression obtained by linear extrapolation in time:

$$
\Delta \mathbf{U}_{i, J L}^{n+1} \approx \Delta \mathbf{U}_{i, J L}^{n}
$$

All subsequent derivative terms, which would necessitate calculating unknowns at more than one $x$ (or $y$ ) line at a time, will be approximated by expressions similar to Eq. (40).

Equations (39) and (40) give the desired relationship between $\Delta \mathrm{U}^{*}$ and $\Delta \mathrm{U}^{n+1}$ at the upper wall:

$$
\Delta \mathbf{U}_{i, J L}^{*}=\Delta \mathbf{U}_{i, J L}^{n+1}+\frac{\Theta \Delta t}{1+\gamma} \mathbf{X}_{i, J L}^{n} \Delta \mathbf{U}_{i, J L}^{n}
$$

\subsection{2. $\Delta \mathbf{U}^{n+1}$ at the Boundary as a Function of $\Delta \mathbf{U}^{n+1}$ at the Interior Grid Points}

$\Delta \mathbf{U}^{n+1}$ at a wall is expressed in terms of $\Delta \mathbf{U}^{n+1}$ at adjacent interior grid points by the following steps:

(i) The pressure at the wall is expressed in terms of $\Delta \mathbf{U}^{n+1}$ at adjacent interior grid points.

(ii) $\Delta \mathrm{U}^{n+1}$ is expressed in terms of the pressure $\Delta P^{n+1}$ at the wall.

The pressure at the wall is expressed in terms of $\Delta \mathbf{U}^{n+1}$ at adjacent interior grid points as follows. At the upper wall, the $y$ momentum equation (Eq. (4)) together with the no-slip condition (Eqs. (29) and (30)) and the assumption of constant transport properties yield

$$
\left(\frac{\partial P}{\partial y}\right)_{i, J L}^{n+1}=\left[-\frac{\partial}{\partial y}\left(\frac{n^{2}}{\rho}\right)+\frac{4}{3} \mu \frac{\partial^{2}}{\partial y^{2}}\left(\frac{n}{\rho}\right)-\frac{1}{3} \mu \frac{\partial^{2}}{\partial x \partial y}\left(\frac{m}{\rho}\right)\right]_{i, J L}^{n+1},
$$

where $m$ and $n$ are defined by Eq. (20). Every term on the right-hand side of the 
above equation is nonlinear with respect to the elements of $\mathbf{U}^{n+1}$. These terms are linearized as shown below.

To avoid the necessity of calculating unknowns at more than one $x$ line at a time, the cross-derivative terms in the above equation are linearized by expressions obtained from linear extrapolation in time:

$$
\frac{1}{3} \mu \frac{\partial^{2}}{\partial x \partial y}\left(\frac{m}{\rho}\right)_{i, J L}^{n+1} \approx \frac{1}{3} \mu \frac{\partial^{2}}{\partial x \partial y}\left(\frac{m}{\rho}\right)_{i, J L}^{n}+\frac{1}{3} \mu \frac{\partial^{2}}{\partial x \partial y} \Lambda\left(\frac{m}{\rho}\right)_{i, J L}^{n} .
$$

The remaining nonlinear terms of Eq. (42) are linearized by truncated Taylor series expansion:

$$
\begin{gathered}
\frac{\partial}{\partial y}\left(\frac{n^{2}}{\rho}\right)_{i, J L}^{n+1}=\frac{\partial}{\partial y}\left[\left(-\frac{n^{2}}{\rho^{2}}\right)^{n} \Delta \rho^{n+1}+\left(\frac{2 n}{\rho}\right)^{n} \Delta n^{n+1}\right]_{i, J L}+\frac{\partial}{\partial y}\left(\frac{n^{2}}{\rho}\right)_{i, J L}^{n}, \quad(44) \\
\frac{4}{3} \mu \frac{\partial^{2}}{\partial y^{2}}\left(\frac{n}{\rho}\right)_{i, J L}^{n+1}=\frac{4}{3} \mu \frac{\partial^{2}}{\partial y^{2}}\left[\left(-\frac{n}{\rho^{2}}\right)^{n} \Delta \rho^{n+1}+\frac{\Delta n^{n+1}}{\rho^{n}}\right]_{i, J L}+\frac{4}{3} \mu \frac{\partial^{2}}{\partial y^{2}}\left(\frac{n}{\rho}\right)_{i, J L}^{n} .
\end{gathered}
$$

By substituting Eqs. (43)-(45) into Eq. (42) and by employing the finite-difference formulas in Table III, the pressure at the wall can be expressed in terms of $\Delta \mathbf{U}^{n+1}$ at adjacent interior grid points in the form

$$
\Delta P_{i, J L}^{n+1}=A_{i}+\mathbf{B}_{i} \Delta \mathbf{U}_{i, J L-1}^{n+1}+\mathbf{C}_{i} \Delta \mathbf{U}_{i, J L-2}^{n+1} .
$$

$A_{i}$ is a scalar and $\mathbf{B}_{i}$ and $\mathbf{C}_{i}$ are five-component row vectors. $A_{i}$ and the elements of $\mathbf{B}_{i}$ and $\mathbf{C}_{i}$ are functions of known quantities $\mathbf{U}^{n}$ and $\mathbf{U}^{n-1}$. The details of $A_{i}$ and the elements of $\mathbf{B}_{i}$ and $\mathbf{C}_{i}$ are irrelevant to the analysis here and are not given, but may be found in Ref. [6].

In order to express $\Delta \mathrm{U}^{n+1}$ in terms of the pressure $\left(\Delta P^{n+1}\right)$ at the wall, it is necessary to relate each element of $\Delta \mathrm{U}^{n+1}(\Delta \rho, \Delta x, \Delta m, \Delta n$, and $\Delta e)$ to $\Delta P^{n+1}$.

At the upper wall, the $x$ - and $y$-components of the velocity are zero. Accordingly, $m$ and $n$ always equal zero so that

$$
\Delta m_{i, J L}^{n+1}=\Delta n_{i, J L}^{n+1}=0
$$

The remaining elements of $\Delta \mathrm{U}^{n+1}$ are related to $\Delta P^{n+1}$ in the following sequence:
(i) Relate $\Delta x^{n+1}$ to $\Delta \rho^{n+1}$.
(ii) Relate $\Delta \rho^{n+1}$ to $\Delta P^{n+1}$.
(iii) Relate $\Delta x^{n+1}$ to $\Delta P^{n+1}$.
(iv) Relate $\Delta e^{n+1}$ to $\Delta P^{n+1}$. 


$$
\begin{aligned}
& \left(\frac{\partial f}{\partial y}\right)_{i, J L} \approx \frac{1}{\Delta y}\left(f_{i, J L}-f_{i, J L-1}\right) \\
& \left(\frac{\partial^{2} f}{\partial y^{2}}\right)_{i, J L} \approx \frac{1}{2 \Delta y^{2}}\left(f_{I, J L}-2 f_{i, J I-1}+f_{i, J-2}\right) \\
& \left(\frac{\partial^{2} f}{\partial x \partial y}\right)_{i, J L} \approx \frac{1}{2 \Delta x \Delta y}\left[\left(f_{i+1, J L}-f_{i-1, J L}\right)-\left(f_{i+1, J L-1}-f_{i-1, J L-1}\right)\right] \\
& \left(\frac{\partial^{2} f}{\partial x \partial y}\right)_{i, J L} \approx \frac{1}{\Delta x \Delta y}\left|\left(f_{2, J L}-f_{1, J L}\right)-\left(f_{2, J L-1}-f_{1, J L-1}\right)\right| \\
& \left.\left(\frac{\partial^{2} f}{\partial x \partial y}\right)_{I L, J L} \approx \frac{1}{\Delta x \Delta y} \mid\left(f_{I L, J L}-f_{I L-1, J L}\right)-\left(f_{I L, J L-1}-f_{I L-1, J L-1}\right)\right] \\
& \left(\frac{\partial f}{\partial x}\right)_{I L, j} \approx \frac{1}{\Delta x}\left(f_{I L, j}-f_{I I-1, j}\right) \\
& \left(\frac{\partial^{2} f}{\partial x^{2}}\right)_{I L, j} \approx \frac{1}{2 \Delta x^{2}}\left(f_{I L, j}-2 f_{I L-1, j}+f_{I L-2, J}\right)
\end{aligned}
$$

where $f$ is any differentiable function.

$\Delta x^{n+1}$ as a function of $\Delta p^{n+1}$. For an impermeable wall, Eq. (31) along with Eq. (20) and the finite-difference formula in Table III yield

$$
X_{A_{i, J L}}^{n+1}=\left(\frac{x}{\rho}\right)_{i, J L}^{n+1} \approx\left(\frac{x}{\rho}\right)_{i, J L-1}^{n+1} \quad \text { or } \quad x_{i, J L}^{n+1} \approx \rho_{i, J L}^{n+1}\left(\frac{x}{\rho}\right)_{i, J L-1}^{n+1} .
$$

By using Eq. (21), the above equation can be rearranged to yield

$$
\Delta x_{i, J L}^{n+1}=\left(x^{n+1}-x^{n}\right)_{i, J L}=\left(\frac{x}{\rho}\right)_{i, J L-:}^{n+1} \Delta \rho_{i, J L}^{n+1}+\rho_{i, J L}^{n} \Delta\left(\frac{x}{\rho}\right)_{i, J L-1}^{n+1} .
$$

The foregoing equation is nonlinear with respect to the elements of $\mathbf{U}^{n+1}$. By linearizing the above equation using truncated Taylor series expansion, we obtain

$$
\Delta x_{i, J L}^{n+1}=\left(\frac{x}{\rho}\right)_{i, J L-1}^{n} \Delta \rho_{i, J L}^{n+1}+\rho_{i, J L}^{n}\left[\left(-\frac{x}{\rho^{2}}\right)^{n} \Delta \rho^{n+1}+\frac{\Delta x^{n+1}}{\rho^{n}}\right]_{i, J L-1} .
$$

$\Delta \rho^{n+1}$ as a function of $\Delta P^{n+1}$. Combining the equation of state (Eq. (16)) with Eqs. (20) and (21) gives 


$$
\begin{aligned}
\Delta P_{i, J L}^{n+1}= & \left(P^{n+1}-P^{n}\right)_{i, J L}=R T_{i, J L}^{n+1}\left[\left(\frac{1}{M_{A}}-\frac{1}{M_{B}}\right) \Delta x+\frac{\Delta \rho}{M_{B}}\right]_{i, J L}^{n+1} \\
& +R\left[\left(\frac{1}{M_{A}}-\frac{1}{M_{B}}\right) x+\frac{\rho}{M_{B}}\right]_{i, J L}^{n}\left(T_{i, J L}^{n+1}-T_{i, J L}^{n}\right) .
\end{aligned}
$$

An expression for the temperature at the wall $T^{n+1}$ is obtained by using Eqs. (32) and (33) and the finite-difference formula in Table III:

$$
T_{i, J L}^{n+1}=T_{i, J L}^{n}=T_{w}
$$

or

$$
T_{i, J L}^{n+1}=T_{i, J L-1}^{n+1}-\frac{q_{w} \Delta y}{\lambda}
$$

Equation (53) is a nonlinear function of the elements of $U^{n+1}$. By linearizing Eq. (53) using truncated Taylor series expansion, we obtain

$$
\begin{aligned}
T_{i, J L-1}^{n+1}= & T_{i, J L-1}^{n}+\left(\frac{\partial T}{\partial \rho}\right)_{i, J L-1}^{n} \Delta \rho_{i, J L-1}^{n+1}+\left(\frac{\partial T}{\partial x}\right)_{i, J L-1}^{n} \Delta x_{i, J L-1}^{n+1} \\
& +\left(\frac{\partial T}{\partial m}\right)_{i, J L-1}^{n} \Delta m_{i, J L-1}^{n+1} \\
& +\left(\frac{\partial T}{\partial n}\right)_{i, J L-1}^{n} \Delta n_{i, J L-1}^{n+1}+\left(\frac{\partial T}{\partial e}\right)_{i, J L-1}^{n} \Delta e_{i, J L-1}^{n+1}-\frac{q_{k} \Delta y}{\lambda}
\end{aligned}
$$

In the following, only the equations for the isothermal wall case will be developed with $T^{n+1}$ given by Eq. (52). Equations (50), (51), and (52) yield

$$
\begin{aligned}
\Delta \rho_{i, J L}^{n+1}= & \left\{\frac{\Delta P_{i, J L}^{n+1}}{R T_{w}}-\rho_{i, J L}^{n}\left(\frac{1}{M_{A}}-\frac{1}{M_{B}}\right)\left[\left(-\frac{x}{\rho^{2}}\right)^{n} \Delta \rho^{n+1}\right.\right. \\
& \left.+\frac{\Delta x^{n+1}}{\rho^{n}}\right]_{i, J L-1}\left\{\left\{\left(\frac{1}{M_{A}}-\frac{1}{M_{B}}\right)\left(\frac{x}{\rho}\right)_{l, J L-1}^{n}+\frac{1}{M_{B}}\right\}^{-1} .\right.
\end{aligned}
$$

$\Delta x^{n+1}$ as a function of $\Delta P^{n+1}$. Equations (50) and (55) yield

$$
\begin{aligned}
\Delta x_{i, J L}^{n+1}= & \left(\frac{x}{\rho}\right)_{i, J L-1}^{n}\left\{\frac{\Delta P_{i, J L}^{n+1}}{R T_{w^{\prime}}}-\rho_{i, J L}^{n}\left(\frac{1}{M_{A}}-\frac{1}{M_{B}}\right)\left[\left(-\frac{x}{\rho^{2}}\right)^{n} \Delta \rho^{n+1}+\frac{\Delta x^{n+1}}{\rho^{n}}\right]_{i, J L-1}\right\} \\
& \times\left\{\left(\frac{1}{M_{A}}-\frac{1}{M_{B}}\right)\left(\frac{x}{\rho}\right)_{i, J L-1}^{n}+\frac{1}{M_{B}}\right\}^{-1} \\
& +\rho_{i, J L}^{n}\left[\left(-\frac{x}{\rho^{2}}\right)^{n} \Delta \rho^{n+1}+\frac{\Delta x^{n+1}}{\rho^{n}}\right]_{i, J L-1}
\end{aligned}
$$


$\Delta e^{n+1}$ as a function of $\Delta P^{n+1}$. Combination of Eqs. (17), (21), (55), and (56) gives

$$
\begin{aligned}
\Delta e_{i, J L}^{n+1}= & \left\{\left[\left(h_{A}^{\circ}-h_{B}^{\circ}\right)+\left(C_{P A}-C_{P B}\right)\left(T_{W}-T_{i}\right)\right]\left(\frac{x}{\rho}\right)_{i, J L-1}^{n}\right. \\
& \left.+\left[\left(h_{B}^{\circ}-C_{P B} T_{i}\right)+T_{W} C_{P B}\right]\right\}\left\{\frac{\Delta P^{n+1}}{R T_{n^{\prime}}}\right. \\
& \left.-\rho_{i, J L}^{n}\left(\frac{1}{M_{A}}-\frac{1}{M_{B}}\right)\left[\left(-\frac{x}{\rho^{2}}\right)^{n} \Delta \rho^{n+1}+\frac{\Delta x^{n+1}}{\rho^{n}}\right]_{i, J L-1}\right\} \\
& \times\left\{\left(\frac{1}{M_{A}}-\frac{1}{M_{B}}\right)\left(\frac{x}{\rho}\right)_{i, J L-1}^{n}+\frac{1}{M_{B}}\right\}^{-1}+\left[\left(h_{A}^{\circ}-h_{B}^{\circ}\right)\right. \\
& \left.+\left(C_{P A}-C_{P B}\right)\left(T_{w^{\prime}}-T_{i}\right)\right] \rho_{i, J L}^{n}\left[\left(-\frac{x}{\rho^{2}}\right)^{n} \Delta \rho^{n+1}+\frac{\Delta x^{n+1}}{\rho^{n}}\right]_{i, J L-1} .
\end{aligned}
$$

By substituting Eqs. (47), (55), (56), and (57) into Eq. (19), $\Delta \mathbf{U}^{n+1}$ can be expressed in terms of $\Delta P^{n+1}$ at the wall in the form

$$
\Delta \mathbf{U}_{i, J L}^{n+1}=\mathbf{D}_{i}+\mathbf{E}_{i} \Delta P_{i, J L}^{n+1}+\mathbf{F}_{i} \Delta \mathbf{U}_{i, J L-1}^{n+1} .
$$

$D_{i}$ and $E_{i}$ are five-component vectors and $F_{i}$ is a five-by-five matrix. The elements of $\mathbf{D}_{i}, \mathbf{E}_{i}$, and $\mathbf{F}_{i}$ are functions of known quantities $\mathbf{U}^{n}$ and $\mathbf{U}^{n-1}$. The details of the elements of $\mathbf{D}_{i}, \mathbf{E}_{i}$, and $\mathbf{F}_{i}$ are not needed in the present analysis and are not given here, but can be found in Ref. [6].

$\Delta \mathbf{U}^{n+1}$ at the wall is related to $\Delta \mathrm{U}^{n+1}$ at interior grid points by substituting Eq. (46) into Eq. (58):

$$
\Delta \mathbf{U}_{i, J L}^{n+1}=\left(\mathbf{D}_{i}+\mathbf{E}_{i} A_{i}\right)+\left(\mathbf{F}_{i}+\mathbf{E}_{i} \mathbf{B}_{i}\right) \Delta \mathbf{U}_{i, J L-1}^{n+1}+\left(\mathbf{E}_{i} \mathbf{C}_{i}\right) \Delta \mathbf{U}_{i, J L-2}^{n+1} .
$$

By combining Eqs. (41) and (59), a relationship between $\Delta \mathrm{U}^{*}$ at the wall and $\Delta \mathrm{U}^{n+1}$ at the two adjacent interior grid points is obtained:

$$
\begin{aligned}
\Delta \mathbf{U}_{i, J L}^{*}= & \left(\mathbf{D}_{i}+\mathbf{E}_{i} A_{i}+\frac{\Theta \Delta t}{1+\gamma} \mathbf{x}_{i, J L}^{n} \Delta \mathbf{U}_{i, J L}^{n}\right)+\left(\mathbf{F}_{i}+\mathbf{E}_{i} B_{i}\right) \Delta \mathbf{U}_{i, J L-1}^{n+1} \\
& +\left(\mathbf{E}_{i} \mathbf{C}_{i}\right) \Delta \mathbf{U}_{i, J L-2}^{n+1} .
\end{aligned}
$$

\subsection{3. $\Delta \mathbf{U}^{*}$ as a Function of $\Delta \mathrm{U}^{n+1}$ at Corresponding Interior Grid Points}

Equation (25) applied at the first and second grid points immediately adjacent to the upper wall becomes

$$
\Delta \mathbf{U}_{i, J L-1}^{*}=\Delta \mathbf{U}_{i, J L-1}^{n+1}+\frac{\Theta \Delta t}{1+\gamma} \mathbf{X}_{i, J L-1}^{n} \Delta \mathbf{U}_{i, J L-1}^{n+1},
$$




$$
\Delta \mathbf{U}_{i, J L-2}^{*}=\Delta \mathbf{U}_{i, J L-2}^{n+1}+\frac{\Theta \Delta t}{1+\gamma} \mathbf{X}_{i, J L-2}^{n} \Delta \mathbf{U}_{i, J L-2}^{n+1}
$$

To avoid the necessity of calculating unknowns at more than one $x$ line at a time, $\Delta \mathbf{U}_{i, J L-1}^{n+1}$ and $\Delta \mathbf{U}_{i, J L-2}^{n+1}$ in the second term of the above equations are approximated by expressions obtained from linear extrapolation in time (e.g., see Eq. (40)). With this approximation, Eqs. (61) and (62) become

$$
\begin{aligned}
& \Delta \mathbf{U}_{i, J L-1}^{n+1}=\Delta \mathbf{U}_{i, J L-1}^{*}-\frac{\Theta \Delta t}{1+\gamma} \mathbf{X}_{i, J L-1}^{n} \Delta \mathbf{U}_{i, J L-1}^{n}, \\
& \Delta \mathbf{U}_{i, J L-2}^{n+1}=\Delta \mathbf{U}_{i, J L-2}^{*}-\frac{\Theta \Delta t}{1+\gamma} \mathbf{X}_{i, J L-2}^{n} \Delta \mathbf{U}_{i, J L-2}^{n} .
\end{aligned}
$$

Substitution of the above two equations into Eq. (60) yields

$$
\Delta \mathbf{U}_{i, J L}^{*}=\mathbf{a}_{i}+\mathbf{b}_{i} \Delta \mathbf{U}_{i, J L-1}^{*}+\mathbf{c}_{i} \Delta \mathbf{U}_{i, J L-2}^{*},
$$

where

$$
\begin{aligned}
\mathbf{a}_{i}= & \mathbf{D}_{i}+\mathbf{E}_{i} \mathbf{A}_{i}+\frac{\Theta \Delta t}{1+\gamma}\left[\mathbf{X}_{i, J L}^{n} \Delta \mathbf{U}_{i, J L}^{n}-\left(\mathbf{F}_{i}+\mathbf{E}_{i} \mathbf{B}_{i}\right) \mathbf{X}_{i, J L-1}^{n} \Delta \mathbf{U}_{i, J L-1}^{n}\right. \\
& \left.-\left(\mathbf{E}_{i} \mathbf{C}_{i}\right) \mathbf{X}_{i, J L-2}^{n} \Delta \mathbf{U}_{i, J L-2}^{n}\right] \\
\mathbf{b}_{i}= & \mathbf{F}_{i}+\mathbf{E}_{i} \mathbf{B}_{i} \\
\mathbf{c}_{i}= & \mathbf{E}_{i} \mathbf{C}_{i}
\end{aligned}
$$

Equation (65) relates $\Delta \mathbf{U}^{*}$ at the wall to $\Delta \mathbf{U}^{*}$ at the adjacent interior grid points. Equation (66) provides the required expressions for $\mathbf{a}_{i}, \mathbf{b}_{i}$, and $\mathbf{c}_{i}$, namely, it gives $\mathbf{a}_{i}$, $\mathbf{b}_{i}$, and $\mathbf{c}_{i}$ as functions of known quantities $\mathbf{U}^{n}$ and $\mathbf{U}^{n-1}$.

\subsection{Expressions for $\Delta \mathrm{U}^{n+1}$ at the Boundary}

For the problem depicted in Fig. 1, expressions for $\Delta \mathbf{U}^{n+1}$ are needed only at the inflow and at the outflow boundaries. Here only an expression for $\Delta \mathbf{U}^{n+1}$ at the outflow boundary $(i=I L, j=1,2, \ldots, J L)$ is derived.

$\Delta \mathbf{U}^{n+1}$ at an outflow boundary is expressed in terms of $\Delta \mathbf{U}^{n+1}$ at adjacent interior grid points by the following steps:

(i) $\Delta \mathrm{U}^{n+1}$ is expressed in terms of the density at the outflow boundary.

(ii) The density at the outflow boundary is expressed in terms of $\Delta \mathrm{U}^{n+1}$ at adjacent interior grid points.

\subsection{1. $\Delta \mathrm{U}^{n+1}$ as a Function of $\Delta \rho^{n+1}$ at the Boundary}

In order to express $\Delta \mathbf{U}^{n+1}$ in terms of density $\left(\Delta \rho^{n+1}\right)$ at the outflow boundary, it 
is necessary to relate each element of $\Delta \mathrm{U}^{n+1}(\Delta \rho, \Delta x, \Delta m, \Delta n$, and $\Delta e)$ to $\Delta \rho^{n+1}$. The elements of $\Delta \mathbf{U}^{n+1}$ are related to $\Delta \rho^{n+1}$ in the following sequence:

(i) Relate $\Delta x^{n+1}$ to $\Delta p^{n+1}$.

(ii) Relate $\Delta m^{n+1}$ to $\Delta \rho^{n+1}$.

(iii) Relate $\Delta n^{n+1}$ to $\Delta \rho^{n+1}$.

(iv) Relate $\Delta e^{n+1}$ to $\Delta \rho^{n+1}$.

$\Delta x^{n+1}, \Delta m^{n+1}$, and $\Delta n^{n+1}$ as functions of $\Delta p^{n+1}$. Equations (35)-(37) together with $\mathrm{Eq}$. (20) and the finite-difference formulas in Table III yield

$$
X_{A_{L L, j}}^{n+1}=\left(\frac{x}{\rho}\right)_{k L, j}^{n+1} \approx 2\left(\frac{x}{\rho}\right)_{l L-1, j}^{n+1}-\left(\frac{x}{\rho}\right)_{l L-2, j}^{n+1}
$$

or

$$
\begin{aligned}
x_{L, j}^{n+1} & \approx \rho_{L L, j}^{n+1}\left[2\left(\frac{x}{\rho}\right)_{I L-1, j}^{n+1}-\left(\frac{x}{\rho}\right)_{l L-2, j}^{n+1}\right], \\
V_{x_{R, j}}^{n+1}=\left(\frac{m}{\rho}\right)_{L L, j}^{n+1} & \approx 2\left(\frac{m}{\rho}\right)_{L L-1, j}^{n+\cdot}-\left(\frac{m}{\rho}\right)_{L L-2, j}^{n+1}
\end{aligned}
$$

or

$$
\begin{aligned}
m_{I L, j}^{n+1} & \approx \rho_{l L, j}^{n+1}\left[2\left(\frac{m}{\rho}\right)_{k-1, j}^{n+1}-\left(\frac{m}{\rho}\right)_{k-2, j}^{n+1}\right], \\
V_{y, j, j}^{n+1}=\left(\frac{n}{\rho}\right)_{l L, j}^{n+1} & \approx 2\left(\frac{n}{\rho}\right)_{k L-1, j}^{n+1}-\left(\frac{n}{\rho}\right)_{k-2, j}^{n+1}
\end{aligned}
$$

or

$$
n_{l L, j}^{n+1} \approx \rho_{l L, j}^{n+1}\left[2\left(\frac{n}{\rho}\right)_{l L-1, j}^{n+1}-\left(\frac{n}{\rho}\right)_{I L-2, j}^{n+1}\right]
$$

The foregoing equations can be rearranged to the form

$$
\begin{aligned}
\Delta Z_{I L, j}^{n+1}=\left(Z^{n+1}-Z^{n}\right)_{L, j}= & {\left[2\left(\frac{Z}{\rho}\right)_{I L-1, j}^{n+1}-\left(\frac{Z}{\rho}\right)_{I L-2, j}^{n+1}\right] \Delta \rho_{I L, j}^{n+1} } \\
& +2 \rho_{k L, j}^{n} \Delta\left(\frac{Z}{\rho}\right)_{I L-1, j}^{n+1}-\rho_{I L, j}^{n} \Delta\left(\frac{Z}{\rho}\right)_{I L-2, j}^{n+1}
\end{aligned}
$$

$z$ denotes either $x, m$, or $n$.

By using truncated Taylor series expansions, Eq. (70) is linearized with respect to the elements of $\mathbf{U}^{n+1}$ giving 


$$
\begin{aligned}
\Delta Z_{I L, j}^{n+1} \approx & {\left[2\left(\frac{Z}{\rho}\right)_{I L-1, j}^{n}-\left(\frac{Z}{\rho}\right)_{I L-2, j}^{n}\right] \Delta \rho_{I L, j}^{n+1} } \\
& +2 \rho_{I L, j}^{n}\left[\left(-\frac{Z}{\rho^{2}}\right)^{n} \Delta \rho^{n+1}+\frac{\Delta Z^{n+1}}{\rho^{n}}\right]_{I L-1, J} \\
& -\rho_{I L, j}^{n}\left[\left(-\frac{Z}{\rho^{2}}\right)^{n} \Delta \rho^{n+1}+\frac{\Delta Z^{n+1}}{\rho^{n}}\right]_{I L-2, J} .
\end{aligned}
$$

$\Delta e^{n+1}$ as a function of $\Delta \rho^{n+1}$. Equations (16), (17), and (34) together with Eq. (20) yield

$$
\begin{aligned}
\Delta e_{I L, j}^{n+1}= & {\left[\left(h_{A}^{\circ}-h_{B}^{\circ}\right)-\left(C_{P A}-C_{P B}\right) T_{i}\right] \Delta x_{I L, j}^{n+1} } \\
& +\left(h_{B}^{\circ}-C_{P B} T_{i}\right) \Delta \rho_{I L, j}^{n+1}+\frac{1}{2} \Delta\left(\frac{m^{2}+n^{2}}{\rho}\right)_{I L, j}^{n+1} \\
& +\frac{P_{b}}{R} \Delta\left[\frac{\left(C_{P A}-C_{P B}\right)\left(\frac{x}{\rho}\right)+C_{P B}}{\left(\frac{1}{M_{A}}-\frac{1}{M_{B}}\right)\left(\frac{x}{\rho}\right)+\frac{1}{M_{B}}}\right]_{L, j}^{n+1} .
\end{aligned}
$$

By using truncated Taylor series expansion, the above equation is linearized with respect to the elements of $\mathbf{U}^{n+1}$ giving

$$
\begin{aligned}
\Delta e_{I L, j}^{n+1} \approx & {\left[\left(h_{A}^{\circ}-h_{B}^{\circ}\right)-\left(C_{P A}-C_{P B}\right) T_{i}\right] \Delta x_{I L, j}^{n+1}+\left(h_{B}^{\circ}-C_{P B} T_{i}\right) \Delta \rho_{I L, j}^{n+1} } \\
& +\frac{1}{2}\left[\frac{2 m^{n}}{\rho^{n}} \Delta m^{n+1}+\frac{2 n^{n}}{\rho^{n}} \Delta n^{n+1}\right]_{I L, j}-\frac{1}{2}\left(\frac{m^{2}+n^{2}}{\rho^{2}}\right)_{I L, j}^{n} \Delta \rho_{I L, j}^{n+1} \\
& +\frac{P_{b}\left(C_{P A}-C_{P B}\right)}{R\left[\left(\frac{1}{M_{A}}-\frac{1}{M_{B}}\right)\left(\frac{x}{\rho}\right)_{I L, j}^{n}+\frac{1}{M_{B}}\right]}\left[\left(-\frac{x}{\rho^{2}}\right)^{n} \Delta \rho^{n+1}+\frac{\Delta x^{n+1}}{\rho^{n}}\right]_{I L, j} \\
& -\frac{P_{b}\left[\left(C_{P A}-C_{P B}\right)\left(\frac{x}{\rho}\right)_{I L, j}^{n}+C_{P B}\right]\left(\frac{1}{M_{A}}-\frac{1}{M_{B}}\right)}{R\left[\left(\frac{1}{M_{A}}-\frac{1}{M_{B}}\right)\left(\frac{x}{\rho}\right)_{I L, j}^{n}+\frac{1}{M_{B}}\right]^{2}} \\
& \times\left[\left(-\frac{x}{\rho^{2}}\right)^{n} \Delta \rho^{n+1}+\frac{\Delta x^{n+1}}{\rho^{n}}\right]_{L L, j} .
\end{aligned}
$$

$\Delta x_{I L, j}^{n+1}$ in the above equation is given by Eq. (71). 
By substituting Eqs. (71) and (73) into Eq. (19), $\Delta \mathbf{U}^{n+1}$ can be expressed in terms of $\Delta \rho^{n+1}$ at the outflow boundary in the form

$$
\Delta \mathbf{U}_{I L, j}^{n+1}=\mathbf{L}_{j} \Delta \rho_{I L, j}^{n+1}+\mathbf{M}_{j} \Delta \mathbf{U}_{I L-1, j}^{n+1}+\mathbf{N}_{j} \Delta \mathbf{U}_{I L-2, j}^{n+1} .
$$

$\mathbf{L}_{j}$ is a five-component vector and $\mathbf{M}_{j}$ and $\mathbf{N}_{j}$ are five-by-five matrices. The elements of $\mathbf{L}_{j}, \mathbf{M}_{j}$, and $\mathbf{N}_{j}$ are functions of known quantities $\mathbf{U}^{n}$ and $\mathbf{U}^{n-1}$. The details of the elements of $\mathbf{L}_{j}, \mathbf{M}_{j}$, and $\mathbf{N}_{j}$ are not needed in the present analysis and are not given here, but can be found in Ref. [6].

\subsection{2. $\Delta \rho^{n+1}$ at the Boundary as a Function of $\Delta \mathbf{U}^{n+1}$ at Interior Grid Points}

The conservation of mass equation (Eq. (1)) applied at the outflow boundary using the notation given by Eq. (20) is

$$
\left(\frac{\partial \rho}{\partial t}+\frac{\partial}{\partial x} m+\frac{\partial}{\partial y} n\right)_{I L, j}^{n+1}=0 .
$$

By replacing the derivatives with respect to $t, x$, and $y$ in the above equation by backward-in-time, one-sided, and central finite-difference formulas, respectively, we obtain

$$
\frac{\Delta \rho_{I L, j}^{n+1}}{\Delta t}+\frac{m_{I L, j}^{n+1}-m_{I L-1, j}^{n+1}}{\Delta x}+\frac{n_{I L, j+1}^{n+1}-n_{I L, j-1}^{n+1}}{2 \Delta y}=0
$$

As before, to avoid the necessity of calculating unknowns at more than one $y$ line at a time, $n_{I L, j+1}^{n+1}$ and $n_{I L, j-1}^{n+1}$ in Eq. (76) are approximated by expressions obtained from linear extrapolation in time:

$$
\begin{aligned}
& n_{I L, j+1}^{n+1} \approx n_{I L, j+1}^{n}+\Delta n_{I L, j+1}^{n}, \\
& n_{I L, j-1}^{n+1} \approx n_{I L, j-1}^{n}+\Delta n_{I L, j-1}^{n} .
\end{aligned}
$$

By combining Eqs. (71), (76)-(78), we obtain the required expression for $\Delta \rho^{n+1}$ :

$$
\begin{aligned}
\Delta \rho_{I L, j}^{n+1}= & \left\{\Delta t\left[\frac{\Delta n_{I L, j-1}^{n}+n_{I L, j-1}^{n}-\left(\Delta n_{I L, j+1}^{n}+n_{I L, j+1}^{n}\right)}{2 \Delta y}+\frac{m_{I L-1, j}^{n}-m_{I L, j}^{n}}{\Delta x}\right]\right. \\
& +\frac{\Delta t}{\Delta x} \Delta m_{I L-1, j}^{n+1}-2 \frac{\Delta t}{\Delta x} \rho_{I L, j}^{n}\left[\left(-\frac{m}{\rho^{2}}\right)^{n} \Delta \rho^{n+1}+\frac{\Delta m^{n+1}}{\rho^{n}}\right]_{I L-1, j} \\
& +\frac{\Delta t}{\Delta x} \rho_{I L, j}^{n}\left[\left(-\frac{m}{\rho^{2}}\right)^{n} \Delta \rho^{n+1}\right. \\
& \left.+\frac{\Delta m^{n+1}}{\rho^{n}}\right]_{I L-2, j}\left\{\left\{1+\frac{\Delta t}{\Delta x}\left[2\left(\frac{m}{\rho}\right)_{I L-1, j}^{n}-\left(\frac{m}{\rho}\right)_{I L-2, j}^{n}\right]\right\}^{-1} .\right.
\end{aligned}
$$


Equation (79) ${ }^{\circ}$ can be written in the form

$$
\Delta \rho_{I L, j}^{n+1}=R_{j}+\mathbf{S}_{j} \Delta \mathbf{U}_{I L-1, j}^{n+1}+\mathbf{T}_{j} \Delta \mathbf{U}_{I L-2, j}^{n+1},
$$

where $R_{j}$ is a scalar and $\mathbf{S}_{j}$ and $\mathbf{T}_{j}$ are five-component row vectors. $R_{j}$ and the elements of $\mathbf{S}_{j}$ and $\mathbf{T}_{j}$ are functions of known quantities $\mathbf{U}^{n}$ and $\mathbf{U}^{n-1}$. The details of $R_{j}, \mathbf{S}_{j}$, and $\mathrm{T}_{j}$ are not needed in the present analysis and are not given here, but can be found in Ref. [6].

Substitution of Eq. (80) into Eq. (74) yields

$$
\Delta \mathbf{U}_{I L, j}^{n+1}=\mathbf{d}_{j}+\mathbf{e}_{j} \Delta \mathbf{U}_{I L-1, j}^{n+1}+\mathbf{f}_{j} \Delta \mathbf{U}_{I L-2, j}^{n+1},
$$

where

$$
\begin{aligned}
& \mathbf{d}_{j}=\mathbf{L}_{j} \mathbf{R}_{j}, \\
& \mathbf{e}_{j}=\mathbf{L}_{j} \mathbf{S}_{j}+\mathbf{M}_{j}, \\
& \mathbf{f}_{j}=\mathbf{L}_{j} \mathbf{T}_{j}+\mathbf{N}_{j} .
\end{aligned}
$$

Equation (81) relates $\Delta \mathbf{U}^{n+1}$ at the outflow boundary to $\Delta \mathbf{U}^{n+1}$ at the adjacent interior grid points. Equation (82) provides the required expressions for $\mathbf{d}_{j}, \mathbf{e}_{j}$, and $\mathbf{f}_{j}$, namely, it gives $\mathbf{d}_{j}, \mathbf{e}_{j}$, and $\mathbf{f}_{j}$ as functions of known quantities $\mathbf{U}^{n}$ and $\mathbf{U}^{n-1}$.

\section{REsults}

To demonstrate the usefulness and the feasibility of the method presented here for deriving implicit boundary conditions for the implicit factored method, numerical solutions were obtained for the flow field inside an axisymmetric piston-cylinder configuration (Fig. 2). The piston-cylinder configuration studied resembles that of a

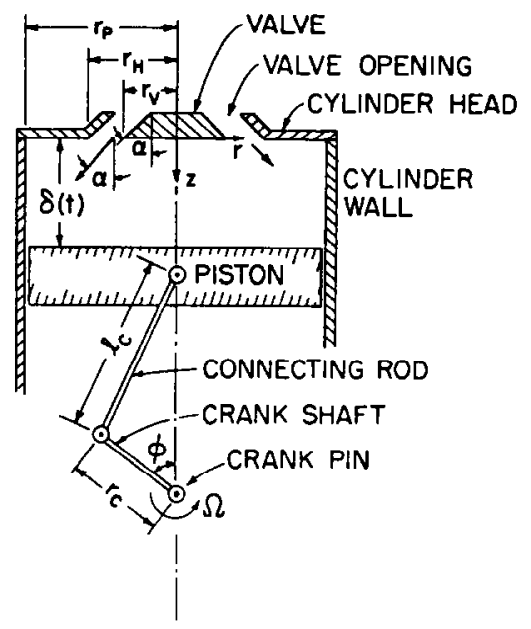

FIG. 2. Geometry of piston-cylinder problem studied. 
reciprocating piston engine. Numerical solutions were generated for the intake and the compression strokes.

Such a flow problem provides a good test of the method for the following reasons:

(i) The flow pattern is complex and includes: (a) sharp temporal and spatial gradients of the dependent variables; (b) separated regions; and (c) recirculating flows.

(ii) The flow may or may not reach a steady-state depending upon whether or not the piston is stationary or moving.

The description of the piston-cylinder problem studied was given elsewhere $[6,14,15]$ and will not be repeated here in detail. Only a brief description of the problem is given to facilitate interpretation of the results.

The piston-cylinder problem analyzed is as follows. A hollow circular cylinder is closed on one end by a flat piston and on the other end by a flat plate (Fig. 2). The piston is connected to a crankshaft through a connecting rod. The piston is driven by
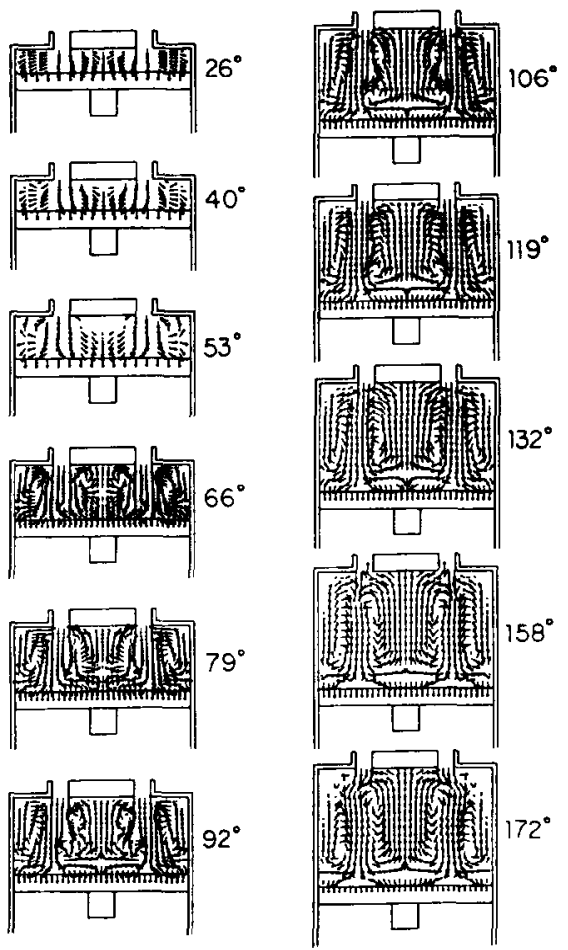

Fig. 3. Flow patterns during the intake stroke as a function of crank angle for two-component gas mixture. 
rotation of the crank shaft about the crankpin. The flat plate has a centrally located annular opening (valve opening) in it which opens instantaneously at the beginning of the intake stroke $(\phi=0)$ and closes instantaneously at the end of the intake stroke $(\phi=\pi)$.

Fluid enters the piston-cylinder configuration described above (henceforth referred to as the cylinder) through the valve opening during the intake stroke. At the valve opening, the entering fluid may have velocity components in the radial $\left(V_{r}\right)$ and axial $\left(V_{z}\right)$ directions, but not in the azimuthal direction. The entering fluid is either a single-component ideal gas or a two-component nonreacting ideal gas mixture. The fluid inside the cylinder at the beginning of the intake stroke is a stagnant, singlecomponent ideal gas.

The continuous domain inside the cylinder was represented by a grid system. The grid size in the axial direction was uniform but changed with time as the piston moved towards or away from the cylinder head. The grid spacing in the radial direction did not change with time, but varied in size to resolve regions where radial derivatives were steep.
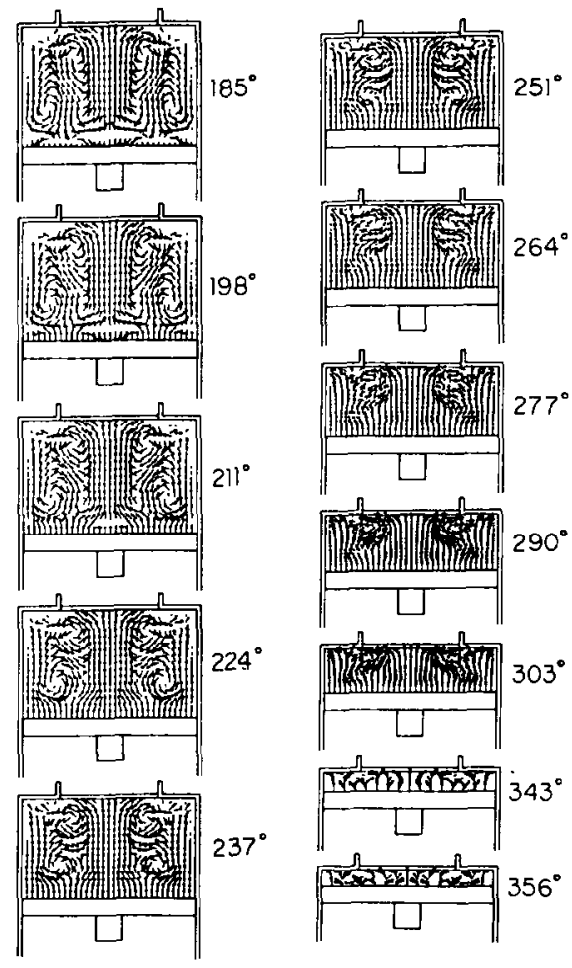

Fig. 4. Flow patterns during the compression stroke as a function of crank angle for twocomponent gas mixture. 
The equations governing the piston-cylinder problem were those in Table I, written in cylindrical coordinates and then transformed to a moving coordinate system corresponding to the grid system described above. For the problems involving a single-component gas, the thermodynamic properties of the gas were taken to be those of air. For the problems involving a two-component gas mixture, the thermodynamic properties were those of air and octane for the two components. The increased mixing due to turbulence was simulated by appropriately chosen effective transport properties. The value of viscosity was taken to be 100 times the viscosity of air at $340^{\circ} \mathrm{K}$ and atmospheric pressure. This is because the effective viscosity for turbulent flows inside spark ignition engine cylinders is roughly this value [16]. The value of the mass diffusivity, $D_{A B}$, and thermal conductivity, $\lambda$, were selected by taking the turbulent Schmidt $\left(\mathrm{Sc}=\mu / \rho D_{A B}\right)$ and $\operatorname{Prandtl}\left(\operatorname{Pr}=\mu C_{p} / \lambda\right)$ numbers to be equal to unity $[17,18]$.

For this problem, implicit boundary conditions were needed at three different types of boundaries, namely: stationary, impermeable wall (cylinder wall, cylinder head, and valve); moving, impermeable wall (piston); and inflow boundary with locally
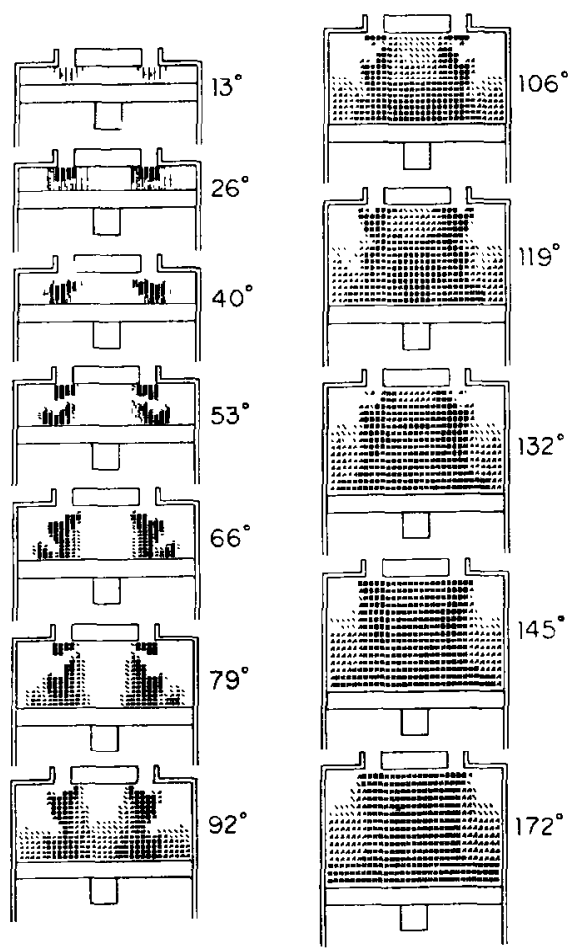

FIG. 5. Distribution of air-to-fuel ratio during the intake stroke as a function of crank angle for twocomponent gas mixture. Darker regions indicate lower air-to-fuel ratios. 
subsonic flow (valve opening). At stationary and moving, impermeable walls, the gas velocity equals the wall velocity, the gas temperature equals the wall temperature, and there is no mass transfer into the wall. At the inflow boundary, the following conditions were specified: the stagnation temperature and stagnation pressure are constant, the flow enters at an angle $\alpha\left(\alpha=\tan ^{-1} V_{r} / V_{z}\right)$, and the mass fraction varies sinusoidally for two-component gas mixtures.

Calculations were performed to determine the gas density, mass fractions, velocity, energy, pressure, and temperature inside the piston-cylinder configuration during the intake and compression strokes for different geometric and operating conditions. All of the results generated were given in Refs. $[6,14,15]$. The results were presented in graphical form showing the flow patterns and mass fractions. Here only typical results are shown illustrating the flow pattern and mass fractions (Figs. 3-6). These representative results demonstrate that the implicit factored method together with the implicit boundary conditions developed in this investigation can be used to obtain solutions to complex flow problems. As was discussed in Refs. [6, 14, 15], the results generated by this method are consistent with the results of previous numerical and experimental studies.
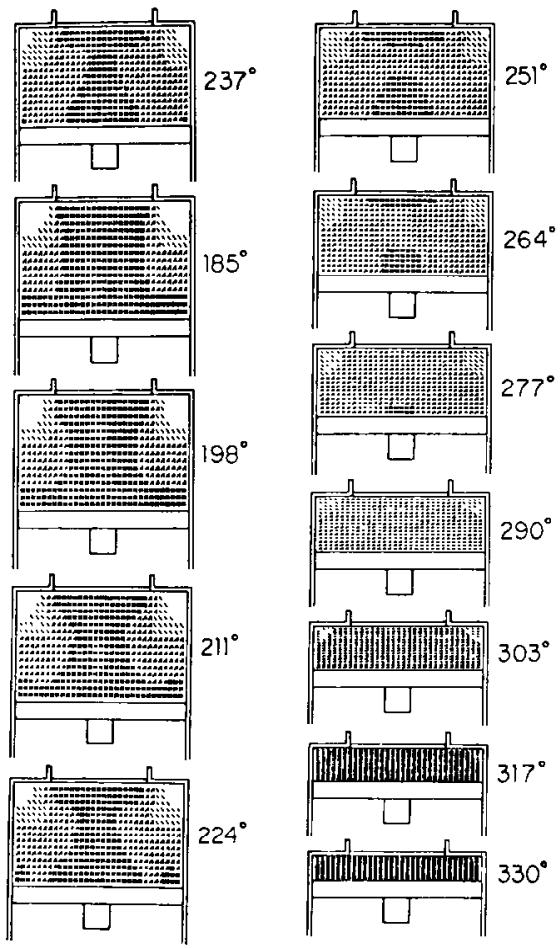

Fig. 6. Distribution of air-to-fuel ratio during the compression stroke as a function of crank angle for two-component gas mixture. Darker regions indicate lower air-to-fuel ratios. 


\section{Stability, Time Step, Grid Sizes, and Computer Time}

\subsection{Stability}

Beam and Warming $|1,2|$ indicated that the implicit factored method employed in the present study should be unconditionally stable. Nevertheless, instability occurs when the time step size exceeds a certain limit. Numerical experiments performed here showed that for the conditions of the present study, the solution was always stable when the time step size $(\Delta t)$ satisfied the expression

$$
\Delta t<60 \Delta W / a_{0} .
$$

$\Delta W$ is the smallest grid size employed in the study and $a_{0}$ is the speed of sound.

Calculations were also performed in which Eqs. (26) and (27) were set equal to zero. This corresponds to using explicit boundary conditions. These numerical experiments showed that for the conditions of the present study, the solution always became unstable when the time step size $(\Delta t)$ exceeded $2 \Delta W / a_{0}$. This result indicates that for the conditions of the present study, the usage of the implicit boundary conditions described here permitted the use of a time step size 30 times larger than that permitted by explicit boundary conditions.

For all of the calculations, fourth-order explicit dissipation terms of the form $\varepsilon_{E} \Delta y^{4} \partial^{4} / \partial y^{4} \mathbf{U}^{n}$ and second-order implicit dissipation terms of the form $\varepsilon_{I} \Delta y^{2} \partial^{2} / \partial y^{2} \Delta \mathrm{U}^{n+1}$ were added to Eqs. (24) and (25). $\varepsilon_{E}$ was set equal to two times $\Delta t$ and $\varepsilon_{I}$ was set equal to two times $\varepsilon_{F}$. These dissipation terms were recommended by Pulliam and Steger [19] to control nonlinear instability. The addition of these dissipation terms do not lower the accuracy of the method based on truncation error analysis.

\subsection{Time Step and Grid Sizes}

As discussed in the previous section, using implicit boundary conditions improves stability and thereby permits the use of larger time step sizes when compared to using explicit boundary conditions. However, in order to obtain sufficiently accurate results, the time step sizes that can actually be used are somewhat lower than that permitted by the stability criterion, Eq. (83). Even by decreasing the time step size to achieve sufficient accuracy, using implicit boundary conditions was still more efficient than using explicit boundary conditions for the conditions of the present study.

The accuracy of numerical solutions, obtained by finite-difference methods. depends on the time step $(\Delta t)$ and grid $(\Delta r$ and $\Delta z$ ) sizes employed. For this problem, mathematical methods do not exist that would provide a good estimate of the accuracy of the numerical solutions as a function of these aforementioned parameters $(\Delta t, \Delta r$, and $\Delta z)$.

In the absence of rigorous accuracy criteria, MacCormack and Lomax [20] suggested that, in order to obtain "accurate" numerical solutions, the time step and grid sizes should at least satisfy the following two conditions: 
(i) The time step size $\Delta t$ should be such that $\Delta t<L / a$, where $L$ is the smallest flow feature length that we wish to resolve and $a$ is the maximum speed with which information is transmitted in the fluid. $a$ is equal to the speed of sound plus the gas velocity.

(ii) In regions where diffusion is important, the grid sizes should be such that the truncation errors introduced by approximating the convection terms (by finitedifference formulas) be much smaller than the values of the diffusion terms.

In order to satisfy the first criterion, the time step size was selected so that the following relation was satisfied:

$$
\Delta t<\left(r_{p} / 2\right) / a_{0} .
$$

$r_{p}$ is the radius of the cylinder and $a_{0}$ is the speed of sound in air.

In order to satisfy the second criterion, the cell Reynolds numbers $\left(\operatorname{Re}_{r}=\rho V, \Delta z / \mu\right.$ and $\left.\operatorname{Re}_{z}=\rho V_{z} \Delta r / \mu\right)$, the cell Peclet numbers $\left(\operatorname{Pé}_{r}=\operatorname{Re}_{r} \operatorname{Pr}\right.$ and $\left.\operatorname{Pé}_{z}=\operatorname{Re}_{z} \operatorname{Pr}\right)$, and the combination of cell Reynolds number and the Schmidt number $\left(\operatorname{Re}_{r} S c\right.$ and $\left.\operatorname{Re}_{z} S c\right)$ at every grid point must be of the order of unity or less $[20,21]$.

Since the Prandtl Pr and Schmidt Sc numbers were taken to be unity (Section 4), only the cell Reynolds number conditions $\left(\operatorname{Re}_{r}<0(1)\right.$ and $\left.\operatorname{Re}_{z}<0(1)\right)$ needed to be satisfied. In this study, $V_{r}, V_{z}$, and $\Delta z$ varied throughout the calculation. Consequently, the cell Reynolds numbers $\left(\operatorname{Re}_{r}\right.$ and $\left.\operatorname{Re}_{z}\right)$ also varied. In this study, $\Delta r$ was $3.125 \times 10^{-3}$ meters and $\Delta z$ varied between $6.25 \times 10^{-4}$ to $8.75 \times 10^{-3}$ meters. The number of grid points used to give the aforementioned grid sizes was $289(17 \times 17)$. The grid points were uniformly distributed inside the cylinder.

For these grid sizes, the cell Reynolds numbers $\left(\operatorname{Re}_{r}\right.$ and $\left.\operatorname{Re}_{z}\right)$ inside the cylinder were less than two. The only exception was in the region of the jet core where, for a short period, the cell Reynolds numbers were as high as ten to twenty. However, in the core of the jet, diffusion is not important and the cell Reynolds number conditions need not be satisfied. Therefore, the grid points employed here resulted in cell Reynolds numbers that satisfied the conditions of $\operatorname{Re}_{r}<0(1)$ and $\operatorname{Re}_{z}<0(1)$ in those regions where diffusion may be important.

One set of calculations was performed in which the effective transport properties $(\mu$ and $\lambda$ ) were reduced by a factor of 100 . These lower value transport properties are those used to compute laminar flow problems. The results of this calculation can be compared with the results obtained under the same geometric and operating conditions except using effective transport properties. This comparison (see Ref. [6]) showed the flow patterns for the two problems to be almost identical when the crank angle $\phi$ was less than 50 degrees. Beyond crank angle $\phi$ of 50 degrees, the flow patterns for these two problems were different because the cell Reynolds numbers $\left(\mathrm{Re}_{r}\right.$ and $\left.\mathrm{Re}_{z}\right)$ for the case using laminar transport properties became very high $(>100)$. The high cell Reynolds number caused oscillations in the solution which eventually rendered the solution meaningless. This effect of the cell Reynolds number has been observed by previous investigators [21-23]. 


\subsection{Computer Time}

With any numerical method, we are concerned with the amount of central processing unit (CPU) time required. In this study, calculations were performed on the IBM $370 / 3033$ computer. The CPU time required per time step using 289 grid points was about $6.89 \times 10^{-3}$ minutes. Calculating the piston-cylinder problems for the intake and compression strokes required about 2900 time steps or 20 minutes.

\section{ACKNOWLEDGMENT}

The authors are grateful to NASA-Lewis Research Center for providing the computer time and the use of their computing facilities.

\section{REFERENCES}

1. R. M. BEAM AND R. F. WARMing, AIAA J. 16 (1978), 393-402.

2. R. F. WARMING and R. M. BeAM, SIAM-ASM Proc. 11 (1978), 85-129.

3. H. C. Yee, R. M. Beam, ANd R. F. Warming, AlAA J. 20 (1982), 1203-1211.

4. R. M. BEAM, R. F. WARMING, AND H. C. YEE, Numerical boundary conditions for unconditionally stable implicit difference approximations of hyperbolic equations, paper presented at SIAM 1981 National Meeting, Troy, N.Y.. June 8-10, 1981.

5. F. A. Williams, "Combustion Theory," Chapter 1. Addison-Wesley, Reading, Mass., 1965.

6. T. I-P. SHIH, "Application of the Implicit Factored Method to Complex Flow Problems." Ph.D. thesis. Dept. of Mechanical Engineering and Applied Mechanics. The University of Michigan. Ann Arbor, 1981.

7. P. D. Thомas, "Boundary Conditions for Implicit Solutions to the Compressible Navier-Stokes Equations in Finite-Computational Domains," AIAA Paper No. 79-1447. American Institute of Aeronautics and Astronautics, 1979.

8. J. Oliger ANd A. SundSTrom, SIAM J. Appl. Math. 35 (1978), 419-446.

9. D. H. Rudy AND J. C. Strikwerda, Comput. \& Fluids 9 (1981), 327-338.

10. H. McDonald and W. R. Briley, J. Comput. Phys. 24 (1977), 372-397.

11. C. K. Chu and A. Sereny, J. Comput. Phys. 15 (1974), 476-491.

12. B. GustafsSon and A. Sundstrom, SIAM J. Appl. Math. 35 (1978), 343-357.

13. B. Gustafsson and H.-O. Kreiss, J. Comput. Phys. 30 (1979), 333-351.

14. T. Shih. G. E. Smith, and G. S. Springer, "Numerical Simulation of the Flow and Fuel-Air Mixing in an Axisymmetric Piston-Cylinder Arrangement," NASA TM 83011, 1982.

15. T. ShiH, G. E. Smith, AND G. S. SpRINGer, Vortex motion in axisymmetric piston-cylinder configurations, submitted.

16. S. C. Johnston, C. W. Robinson, W. S. Rorke, J. R. Smith, and P. O. Witze, "Application of Laser Diagnostics to an Injected Engine," SAE Paper No. 790092, Society of Automotive Engineers, 1979.

17. B. E. Launder and D. B. Spalding, "Lectures in Mathematical Models of Turbulence," Chapter 3, Academic Press, London/New York, 1972.

18. A. J. Reynolds, Internat. J. Heat Mass Transfer 18 (1975), 1055-1069.

19. T. H. Pulliam and J. L. Steger, aIAA J. 18 (1980), 159-167.

20. R. W. MACCORMACK AND H. Lomax, Numerical solution of compressible viscous flows, in "Annual Review of Fluid Mechanics," pp. 289-316, Annual Reviews Inc., Palo Alto, Calif., 1979.

21. P. J. RoAche, "Computational Fluid Dynamics," Hermosa Publishers, Albuquerque, N. Mex.. 1976. 
22. M. D. Griffin, J. D. Anderson, Jr., and R. Diwaker, AIAA J. 14 (1976), 1665-1666.

23. M. D. GrifFiN, "Numerical Solutions for Two- and Three-Dimensional Non-Reacting Flowfields in an Internal Combustion Engine," Ph.D. thesis, University of Maryland, College Park, Maryland, 1977. 